\title{
Conservation importance of the melliferous plants of the Bulgarian flora
}

\author{
ALEXANDER TASHEV \\ EVGENIA PANCHEVA
}

ТАШЕВ О., ПАНЧЕВА $Є$., 2011: Природоохоронне значення медоносних рослин Болгарської флори. Чорноморськ. бот. ж., Т. 7, № 2: 103-112.

\begin{abstract}
В статті представлена класифікація 1010 видів, які належать до 296 родів та 84 родин диких медоносних рослин. Особлива увага приділялася характеристиці природоохоронного значення цих рослин на національному та міжнародному рівні. Серед них 201 вид, які належать до 71 роду та 36 родин та були визнані, як такі, що мають певний природоохоронний статус в Болгарії та Європі. Більшість 3 них $є$ ендеміками Болгарії i/aбо Балканського півострова i охороняються згідно 3 національним болгарським законодавством або міжнародними конвенціями ратифікованими Болгарією. Результати показують, що суттєва частина медоносних видів рослин болгарської флори має високу природоохоронну цінність.
\end{abstract}

Ключові слова: Болгарія, флора, медоносні рослини, природоохоронна цุінність

TASHev A., PAncheva E., 2011: Conservation importance of the melliferous plants of the Bulgarian flora. Chornomors 'k. bot. $z$, Vol. 7, № 2: 103-112.

The paper presents a classification of 1010 species, belonging to 296 genera and 84 families of wild melliferous plants. A particular attention is given to the characteristics of the conservational importance of these plants at national and international level. Two hundred and one of these species belonging to 71 genera and 36 families were considered to have some conservational status in Bulgaria and Europe. Many of them are endemics for Bulgaria and/or Balkan Peninsula and are protected by the national Bulgarian legislation or by international conventions ratified by Bulgaria. The results indicate that substantial part of melliferous plant species of the Bulgarian flora is of high conservation value.

Key words: Bulgaria, flora, melliferous plants, conservation value

ТАШЕВ А., ПАНЧЕВА Е., 2011: Природоохранное значение медоносных растений Болгарской флоры. Черноморск. бот. ж., Т. 7, № 2: 103-112.

В статье представлена классификация 1010 видов, которые принадлежат к 296 родам и 84 семействам диких медоносных растений. Особое внимание уделялось характеристике природоохранного значения этих растений на национальном и международном уровне. Среди них 201 вид, которые принадлежат к 71 роду и 36 семействам и были признаны, как имеющие определенный природоохранный статус в Болгарии и Европе. Большинство из них являются эндемиками Болгарии и/или Балканского полуострова и охраняются согласно с национальным болгарским законодательством или международными конвенциями ратифицированными Болгарией. Результаты указывают, что значительная часть медоносных видов растений болгарской флоры имеет высокую природоохранную ценность.

Ключевые слова: Болгария, флора, медоносные растения, природоохранная ценность

Melliferous plants are these, whose flowers are natural food source for honey-bee (Apis mellifera L.). Based on critical analysis of literature data, it was established that wild

(C) А. Ташева, Е. Панчева

Чорноморськ. бот. ж., Т. 7, № 2: 103-112. 
melliferous plants in Bulgaria are represented by 1010 species from 296 genera, 84 families and two phyla [TASHEV, PANCHEVA, 2010]. This represents $25,3 \%$ of the species, $31,7 \%$ of the genera and 54,9\% of the families of the Bulgarian flora [TASHEV, 2008].

Being an integral part of the plant kingdom, melliferous plants are charactrerized by different life-histories, different ecology and conservation status. The assessment of their conservation status is an important part of the modern concepts and practices for sustainable use of plant resources and conservation of plant diversity. Therefore, the aim of the present study was to characterize the conservation importance of the wild melliferous plants of Bulgarian flora. National and international documents were used for this assessment, with particular reference to the endemic taxa.

\section{Material and Methods}

In the determination of the national conservation status of the melliferous plants from the Bulgarian flora, we used the Red Data Book of Bulgaria, Volume I - Plants [VELCHEV, 1984], Red Data Book of Bulgaria, Volume I - Plant and Fungi (Peev in press). We also used the national legislation treating the legal protection of the plant species [BIODIVERSITY ACT; ANONYMOUS, 2002]. Determination of the international status of the analyzed species is based on the Red List of Endangered Plants of IUCN [WALTER, GILlETt, 1998] as well as on the "List of Rare, Endangered and Endemic Plants of Europe" [LUCAS, 1983]. We also used the CITES [AnONymous, 1973] and the Convention on the Conservation of European Wildlife and Natural Habitats called also Bern Convention [ANONyMOUS, 1979]. The Balkan and Bulgarian endemics were determined following Assyov and Petrova [2006].

\section{Results and Discussion}

Table 1 shows that considerable part of meliferous plants of Bulgarian flora is important from conservation point of view and the species are included in the Red Data Book of Bulgaria, Volume I - Plants (Velchev 1984). They are totally 112 species, or 11,1\% of all species of this group. Among them with category "Rare" (R) are 78 species, and with category "Endangered" (EN) - 34 species. In percents, compared to the whole number of melliferous plants of the Bulgarian flora, the distribution is as follows: 7,7\% "Rare" and 3,4\% "Endangered". When compared to the number of all wild melliferous plants, included in the Red Data Book of Bulgaria, 69,6\% are "Rare" and 30,4\% are "Endangered".

In the new edition of the Red Data Book of Bulgaria, Volume I - Plants and Fungi (Peev in press), the number of melliferous plants is reduced to 74 species $(7,4 \%)$. This could be explained by the fact that many of the species included in the first edition of the Red Data Book (Velchev 1984) had category "Rare", which does not exist in the new edition. This is because it considers only IUCN threatened categories, i.e. "Extinct", "Critically Endangered", and "Endangered". A few species labeled as "Rare" in Velchev (1984) were included with the category "Vulnerable" in the Red List of Bulgarian vascular plants (Petrova and Vladimirov 2009). The new edition of the Red Data Book of Bulgaria includes 43 species with the category "Endangered" (EN), which is $4,3 \%$ of all wild melliferous plants; "Critically Endangered" (CR) are $26(2,6 \%)$ species, and "Vulnerable" (VU) are $5(0,5 \%)$ species.

Comparison of the plant taxa in the two Red Data Book editions shows that 50 melliferous species are present in both of them. This means that in the period after the publication of the first edition (more than 25 years), 24 wild melliferous species have acquired conservation importance. Eighteen species were transferred from category "Rare" to category "Endangered"; 11 species from category "Rare" to category "Critically Endangered"; 2 species from category "Rare" to category "Vulnerable", and 5 species from category "Endangered" to category "Critically Endangered". This unequivocally means that the populations' status of the rare melliferous plants is getting worse. 
Conservation status of melliferous plants of Bulgarian flora

Table 1

\begin{tabular}{|c|c|c|c|c|c|c|c|c|c|c|c|}
\hline \multirow{3}{*}{ Family/Species } & \multicolumn{7}{|c|}{ Conservation importance } & \multicolumn{3}{|c|}{ Measures taken } & \multirow{3}{*}{ 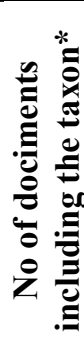 } \\
\hline & \multirow{2}{*}{ 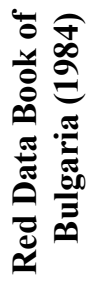 } & \multirow{2}{*}{ 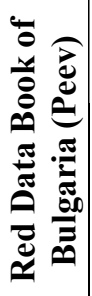 } & \multirow{2}{*}{ 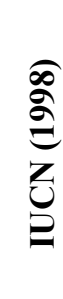 } & \multicolumn{2}{|c|}{$\begin{array}{l}\text { Eur. List, } \\
\text { (1983) }\end{array}$} & \multirow{2}{*}{ 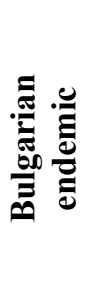 } & \multirow{2}{*}{ 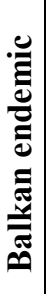 } & \multirow{2}{*}{ 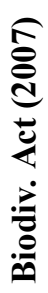 } & \multirow{2}{*}{ 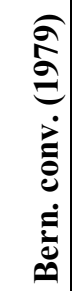 } & \multirow{2}{*}{ 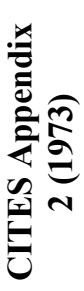 } & \\
\hline & & & & $\bigcup_{\infty}^{0}$ & D & & & & & & \\
\hline 1 & 2 & 3 & 4 & 5 & 6 & 7 & 8 & 9 & 10 & 11 & 12 \\
\hline \multicolumn{12}{|l|}{ Acanthaceae } \\
\hline Acanthus spinosus L. & $\mathrm{R}$ & EN & - & - & - & - & - & + & - & - & 3 \\
\hline $\begin{array}{l}\text { Acanthus balcanicus Heywood et } \\
\text { Richardson }\end{array}$ & - & - & - & - & - & - & + & - & - & - & 1 \\
\hline \multicolumn{12}{|l|}{ Alliaceae } \\
\hline Allium angulosum L. & $\mathrm{R}$ & $\mathrm{CR}$ & - & - & - & - & - & + & - & - & 3 \\
\hline Allium montanum F. W. Schmidt & $\mathrm{EN}$ & - & - & - & - & - & - & + & - & - & 2 \\
\hline Allium rhodopaeum Velen. & - & - & - & - & - & + & - & - & - & - & 1 \\
\hline \multicolumn{12}{|l|}{ Amaryllidaceae } \\
\hline Galanthus elwesii Hook. f. & $\mathrm{EN}$ & EN & - & - & - & - & - & + & - & + & 4 \\
\hline Galanthus nivalis L. & $\mathrm{EN}$ & $\mathrm{EN}$ & - & - & - & - & - & + & - & + & 4 \\
\hline Leucojum aestivum L. & EN & - & - & - & - & - & - & - & - & - & 1 \\
\hline \multicolumn{12}{|l|}{ Apiaceae (Umbelliferae) } \\
\hline Anethum graveolens L. & $\mathrm{R}$ & EN & - & - & - & - & - & - & - & - & 2 \\
\hline $\begin{array}{l}\text { Heracleum angustisectum (Stoj. et } \\
\text { Acht.) Peev }\end{array}$ & $\mathrm{R}$ & - & - & - & - & + & - & - & - & - & 2 \\
\hline Heracleum verticillatum Panč. & - & - & - & - & - & - & + & - & - & - & 1 \\
\hline \multicolumn{12}{|l|}{ Asteraceae (Compositae) } \\
\hline Hieracium ančevii Szelag & - & - & - & - & - & + & - & - & - & - & 1 \\
\hline $\begin{array}{l}\text { Hieracium asenovgradense Jasiewicz } \\
\text { \& Pawl. }\end{array}$ & - & - & - & - & - & + & - & - & - & - & 1 \\
\hline $\begin{array}{l}\text { Hieracium belogradčense T. Georg. } \\
\text { \& Kitanov }\end{array}$ & - & $\mathrm{CR}$ & - & - & - & + & - & + & - & - & 3 \\
\hline Hieracium divergens Naeg. \& Peter & - & - & - & - & - & + & - & - & - & - & 1 \\
\hline Hieracium dolopicum Freyn \& Sint. & - & - & - & - & - & - & + & - & - & - & 1 \\
\hline Hieracium ferdinandii-regis Zahn & - & - & - & - & - & + & - & - & - & - & 1 \\
\hline $\begin{array}{l}\text { Hieracium gregorii-bakurianii S. } \\
\text { Bräut. }\end{array}$ & - & - & - & - & - & + & - & - & - & - & 1 \\
\hline Hieracium heldreichii Boiss. & - & - & - & - & - & - & + & - & - & - & 1 \\
\hline Hieracium heterogynum (Froel.) Gut. & - & - & - & - & - & - & + & - & - & - & 1 \\
\hline Hieracium kittaniae Vladimirov & - & EN & - & - & - & + & - & - & - & - & 2 \\
\hline Hieracium marmoreum Panč. & - & - & - & - & - & - & + & - & - & - & 1 \\
\hline Hieracium mattffeldianum Zahn & - & - & - & - & - & + & - & - & - & - & 1 \\
\hline $\begin{array}{l}\text { Hieracium merxmulleranum } \mathrm{S} \text {. } \\
\text { Bräut. }\end{array}$ & - & - & - & - & - & + & - & - & - & - & 1 \\
\hline Hieracium naegelianum Panč. & - & - & - & - & - & - & + & - & - & - & 1 \\
\hline $\begin{array}{l}\text { Hieracium nipholasum T. Georg. \& } \\
\text { Zahn }\end{array}$ & - & - & - & - & - & + & - & - & - & - & 1 \\
\hline
\end{tabular}




\begin{tabular}{|c|c|c|c|c|c|c|c|c|c|c|c|}
\hline 1 & 2 & 3 & 4 & 5 & 6 & 7 & 8 & 9 & 10 & 11 & 12 \\
\hline Hieracium olympicum Boiss. & - & - & - & - & - & - & + & - & - & - & 1 \\
\hline Hieracium ossaeum Zahn & - & - & - & - & - & - & + & - & - & - & 1 \\
\hline Hieracium pannosum Boiss. & - & - & - & - & - & - & + & - & - & - & 1 \\
\hline $\begin{array}{l}\text { Hieracium petrovae Vladimirov \& } \\
\text { Szelag }\end{array}$ & - & - & - & - & - & + & - & - & - & - & 1 \\
\hline Hieracium pilosissimum Friv. & - & - & - & - & - & - & + & - & - & - & 1 \\
\hline $\begin{array}{l}\text { Hieracium pirinicola T. Georg. \& } \\
\text { Zahn }\end{array}$ & - & - & - & - & - & + & - & - & - & - & 1 \\
\hline Hieracium pseuderiopus Zahn & - & - & - & - & - & - & + & - & - & - & 1 \\
\hline $\begin{array}{l}\text { Hieracium scardicum Bornm. \& } \\
\text { Zahn }\end{array}$ & - & - & - & - & - & - & + & - & - & - & 1 \\
\hline Hieracium sericophyllum Nejčeff & - & - & - & - & - & - & + & - & - & - & 1 \\
\hline Hieracium stefanofii Zahn & $\mathrm{R}$ & - & - & - & - & + & - & - & - & - & 2 \\
\hline Hieracium tommassinii Rchb. & - & - & - & - & - & - & + & - & - & - & 1 \\
\hline Hieracium urumoffii Nejčeff \& Zahn & - & - & - & - & - & + & - & - & - & - & 1 \\
\hline Hieracium villosum L. & - & EN & - & - & - & - & - & - & - & - & 1 \\
\hline Hieracium virosum Pall. & - & $\mathrm{EN}$ & - & - & - & - & - & - & - & - & 1 \\
\hline \multicolumn{12}{|l|}{ Berberidaceae } \\
\hline $\begin{array}{l}\text { Epimedium pubigerum (DC.) Morren } \\
\text { \& Decne }\end{array}$ & $\mathrm{R}$ & - & - & - & - & - & - & + & - & - & 2 \\
\hline \multicolumn{12}{|l|}{ Boraginaceae } \\
\hline Anchusa davidovii Stoj. & - & $\mathrm{CR}$ & - & - & - & + & - & + & - & - & 3 \\
\hline Anchusa hybrida Ten. & $\mathrm{R}$ & - & - & - & - & - & - & - & - & - & 1 \\
\hline $\begin{array}{l}\text { Buglossoides arvensis (L.) I. M. } \\
\text { Johnst. }\end{array}$ & $\mathrm{R}$ & - & - & - & - & -- & - & - & - & - & 1 \\
\hline $\begin{array}{l}\text { Buglossoides grandulosa (Velen.) R. } \\
\text { Fernandes }\end{array}$ & $\mathrm{R}$ & - & $\mathrm{R}$ & $\mathrm{V}$ & $\mathrm{V}$ & - & - & + & - & - & 4 \\
\hline $\begin{array}{l}\text { Buglossoides sibthorpiana (Griseb.) } \\
\text { Czer. }\end{array}$ & $\mathrm{R}$ & - & - & - & - & - & - & - & - & - & 1 \\
\hline Echium plantagineum L. & $\mathrm{R}$ & - & - & - & - & - & - & - & - & - & 1 \\
\hline Echium russicum J. F. Gmel. & - & $\mathrm{VU}$ & - & - & - & - & - & + & - & - & 2 \\
\hline Myosotis aspera Velen. & - & - & - & - & - & + & - & - & - & - & 1 \\
\hline $\begin{array}{l}\text { Myosotis jordanovii N. Andreev \& } \\
\text { Peev }\end{array}$ & - & - & - & - & - & + & - & - & - & - & 1 \\
\hline $\begin{array}{l}\text { Myosotis macedonica } \text { Velen. \& } \\
\text { Charrel }\end{array}$ & - & - & - & - & - & - & + & - & - & - & 1 \\
\hline $\begin{array}{l}\text { Myosotis orbelica (Velen.) Peev \& } \\
\text { N. Andreev }\end{array}$ & - & - & - & - & - & + & - & - & - & - & 1 \\
\hline Myosotis rhodopea Velen. & - & - & - & - & - & + & - & - & - & - & 1 \\
\hline Myosotis suaveolens Waldst \& Kit. & - & - & - & - & - & - & + & - & - & - & 1 \\
\hline Onosma heterophylla Griseb. & $\mathrm{R}$ & - & - & - & - & - & - & - & - & - & 1 \\
\hline Onosma thracica Velen. & - & - & - & $\mathrm{nt}$ & - & - & + & - & - & - & 2 \\
\hline Onosma rhodopea Velen. & $\mathrm{EN}$ & EN & $\mathrm{R}$ & $\mathrm{I}$ & $\mathrm{R}$ & - & + & + & - & - & 6 \\
\hline Symphytum tauricum Willd. & $\mathrm{R}$ & EN & - & - & - & - & - & - & - & - & 2 \\
\hline Trachystemon orientalis (L.) G. Don & $\mathrm{R}$ & - & - & - & - & - & - & - & - & - & 1 \\
\hline \multicolumn{12}{|l|}{ Brassicaceae (Cruciferae) } \\
\hline $\begin{array}{l}\text { Alyssum cuneifolium Ten. var. } \\
\text { pirinicum Stoj. \& Acht. }\end{array}$ & $\mathrm{R}$ & EN & - & - & - & - & - & + & - & - & 3 \\
\hline Alyssum pulvinare Velen. & $\mathrm{R}$ & - & - & - & - & - & - & - & - & - & 1 \\
\hline
\end{tabular}




\begin{tabular}{|c|c|c|c|c|c|c|c|c|c|c|c|}
\hline 1 & 2 & 3 & 4 & 5 & 6 & 7 & 8 & 9 & 10 & 11 & 12 \\
\hline Alyssum stribrnyi Velen. & $\mathrm{R}$ & - & - & - & - & - & - & + & - & - & 2 \\
\hline $\begin{array}{l}\text { Matthiola odoratissima (M. Bieb.) R. } \\
\mathrm{Br} \text {. }\end{array}$ & EN & $\mathrm{CR}$ & - & V & V & - & - & + & - & - & 4 \\
\hline \multicolumn{12}{|l|}{ Caesalpiniaceae } \\
\hline Cercis siliquastrum L. & $\mathrm{R}$ & - & - & - & - & - & - & - & - & - & 1 \\
\hline \multicolumn{12}{|l|}{ Campanulaceae } \\
\hline Campanula cochlearifolia Lam. & - & EN & - & - & - & - & - & - & - & - & 1 \\
\hline Campanula euxina (Velen.) Ančev & - & EN & - & - & - & + & - & + & - & - & 3 \\
\hline $\begin{array}{l}\text { Campanula jordanovii Ančev \& } \\
\text { Kovanda }\end{array}$ & - & VU & - & - & - & - & + & + & - & - & 3 \\
\hline Campanula lanata Friv. & - & VU & $\mathrm{R}$ & - & - & - & + & + & + & - & 5 \\
\hline Campanula latifolia $\mathrm{L}$. & $\mathrm{R}$ & EN & - & - & - & - & - & + & - & - & 3 \\
\hline Campanula moesiaca Velen. & - & - & - & - & - & - & + & - & - & - & 1 \\
\hline Campanula orphanidea Boiss. & - & EN & - & - & - & - & + & + & - & - & 3 \\
\hline Campanula patula $\mathrm{L}$. & - & - & - & - & - & - & - & - & + & - & 1 \\
\hline Campanula scutellata Griseb. & - & - & - & - & - & - & + & - & - & - & 1 \\
\hline Campanula sparsa Friv. & - & - & - & - & - & - & + & - & - & - & 1 \\
\hline Campanula thyrsoides L. & - & EN & - & - & - & - & - & - & - & - & 1 \\
\hline $\begin{array}{l}\text { Campanula transsilvanica Schur ex } \\
\text { Andrae }\end{array}$ & $\mathrm{R}$ & EN & $\mathrm{R}$ & $\mathrm{R}$ & $\mathrm{I}$ & - & - & + & - & - & 5 \\
\hline $\begin{array}{l}\text { Campanula trojanensis Kovanda \& } \\
\text { Ančev }\end{array}$ & - & - & - & - & - & + & - & - & - & - & 1 \\
\hline Campanula velebitica Borbas & - & - & - & - & - & - & + & - & - & - & 1 \\
\hline Campanula versicolor Andrews & EN & EN & - & - & - & - & - & + & - & - & 3 \\
\hline \multicolumn{12}{|l|}{ Cistaceae } \\
\hline Cistus salvifolius $\mathrm{L}$. & $\mathrm{R}$ & EN & - & - & - & - & - & + & - & - & 3 \\
\hline \multicolumn{12}{|l|}{ Elaeagnaceae } \\
\hline Hippophae rhamnoides L. & EN & $\mathrm{CR}$ & - & - & - & - & - & + & - & - & 3 \\
\hline \multicolumn{12}{|l|}{ Ericaceae } \\
\hline Calluna vulgaris (L.) Hull & $\mathrm{R}$ & - & - & - & - & - & - & + & - & - & 2 \\
\hline Erica arborea L. & $\mathrm{R}$ & - & - & - & - & - & - & + & - & - & 2 \\
\hline Rhododendron ponticum L. & EN & - & - & - & - & - & - & + & - & - & 2 \\
\hline Vaccinium arctostaphylos L. & EN & EN & - & $\mathrm{R}$ & $\mathrm{R}$ & - & - & + & + & - & 5 \\
\hline \multicolumn{12}{|l|}{ Fabaceae (Papilionaceae) } \\
\hline Anthyllis aurea Weld. & - & - & - & - & - & - & + & - & - & - & 1 \\
\hline $\begin{array}{l}\text { Chamaecytisus danubialis (Velen.) } \\
\text { Rothm. }\end{array}$ & $\mathrm{R}$ & - & - & - & - & - & - & - & - & - & 1 \\
\hline $\begin{array}{l}\text { Chamaecytisus absimthioides (Janka) } \\
\text { Kuzm. }\end{array}$ & - & - & - & - & - & - & + & - & - & - & 1 \\
\hline $\begin{array}{l}\text { Chamaecytisus calcareus (Velen.) } \\
\text { Kuzm. }\end{array}$ & - & - & - & - & - & - & + & - & - & - & 1 \\
\hline $\begin{array}{l}\text { Chamaecytisus jankae (Velen.) } \\
\text { Rothm. }\end{array}$ & - & - & - & - & - & - & + & - & - & - & 1 \\
\hline $\begin{array}{l}\text { Chamaecytisus frivaldszkyanus } \\
\text { (Deg.) Kuzm. }\end{array}$ & $\mathrm{R}$ & EN & - & - & - & + & - & - & - & - & 3 \\
\hline $\begin{array}{l}\text { Chamaecytisus kovačevii (Velen.) } \\
\text { Rothm. }\end{array}$ & $\mathrm{R}$ & EN & - & $\mathrm{V}$ & - & + & - & + & - & - & 5 \\
\hline $\begin{array}{l}\text { Chamaecytisus neičeffii (Urum.) } \\
\text { Rothm. }\end{array}$ & $\mathrm{R}$ & - & VU & E & - & + & - & + & - & - & 5 \\
\hline
\end{tabular}




\begin{tabular}{|c|c|c|c|c|c|c|c|c|c|c|c|}
\hline 1 & 2 & 3 & 4 & 5 & 6 & 7 & 8 & 9 & $\mathbf{1 0}$ & 11 & 12 \\
\hline $\begin{array}{l}\text { Chamaecytisus ratisbonensis } \\
\text { (Schaeff.) Rothm. }\end{array}$ & EN & - & - & - & - & - & - & + & - & - & 2 \\
\hline Genista germanica L. & - & $\mathrm{CR}$ & - & - & - & - & - & + & - & - & 2 \\
\hline Genista pilosa L. & $\mathrm{EN}$ & EN & - & - & - & - & - & + & - & - & 3 \\
\hline Genista rumelica Velen. & - & - & - & - & - & - & + & - & - & - & 1 \\
\hline Genista subcapitata Panč. & - & - & - & - & - & - & + & - & - & - & 1 \\
\hline Lathyrus grandiflorus Sibth. et Sm. & $\mathrm{R}$ & EN & - & - & - & - & - & - & - & - & 2 \\
\hline Lupinus albus L. & $\mathrm{R}$ & - & - & - & - & - & - & - & - & - & 1 \\
\hline Ononis adenotricha Boiss. & $\mathrm{R}$ & - & - & - & - & - & - & - & - & - & 1 \\
\hline Ononis repens L. & $\mathrm{R}$ & $\mathrm{CR}$ & - & - & - & - & - & + & - & - & 3 \\
\hline Trifolium affine C. Presl. & - & - & - & $\mathrm{R}$ & $\mathrm{R}$ & - & - & - & - & - & 1 \\
\hline Trifolium dalmaticum Vis. & - & - & - & - & - & - & + & - & - & - & 1 \\
\hline Trifolium heldreichianum Hausskn. & - & - & - & - & - & - & + & - & - & - & 1 \\
\hline Trifolium pignantii Fauche et Chaub. & - & - & - & - & - & - & + & - & - & - & 1 \\
\hline Trifolium trichopterum Panč. & - & - & - & - & - & - & + & - & - & - & 1 \\
\hline Trifolium globosum L. & $\mathrm{R}$ & - & - & - & - & - & - & - & - & - & 1 \\
\hline Trifolium spumosum L. & $\mathrm{R}$ & - & - & - & - & - & - & - & - & - & 1 \\
\hline Trifolium ligusticum Balb. ex Loisel & EN & EN & - & - & - & - & - & - & - & - & 2 \\
\hline Trifolium phleoides Pourr. ex Willd. & $\mathrm{EN}$ & $\mathrm{CR}$ & - & - & - & - & - & - & - & - & 2 \\
\hline Trifolium physodes Stev. ex M. B. & - & $\mathrm{CR}$ & - & - & - & - & - & - & - & - & 1 \\
\hline Trifolium rubens $\mathrm{L}$. & EN & $\mathrm{CR}$ & - & - & - & - & - & - & - & - & 2 \\
\hline Trifolium spumosum L. & - & $\mathrm{CR}$ & - & - & - & - & - & - & - & - & 1 \\
\hline Trifolium squamosum L. & EN & $\mathrm{CR}$ & - & - & - & - & - & - & - & - & 2 \\
\hline Trifolium squarrosum L. & EN & $\mathrm{CR}$ & - & - & - & - & - & - & - & - & 2 \\
\hline Trifolium velenovskyi Vandas & - & - & - & - & - & - & + & - & - & - & 1 \\
\hline \multicolumn{12}{|l|}{ Fagaceae } \\
\hline Castanea sativa Mill. & $\mathrm{EN}$ & EN & - & - & - & - & - & - & - & - & 2 \\
\hline \multicolumn{12}{|l|}{ Geraniaceae } \\
\hline Erodium absinthoides Willd. & $\mathrm{R}$ & $\mathrm{EN}$ & - & - & - & - & + & + & - & - & 4 \\
\hline Erodium hoefftianum C. A. Meyer & $\mathrm{R}$ & - & - & - & - & - & - & - & - & - & 1 \\
\hline \multicolumn{12}{|l|}{ Grossulariaceae } \\
\hline Ribes nigrum L. & $\mathrm{R}$ & $\mathrm{CR}$ & - & - & - & - & - & + & - & - & 3 \\
\hline \multicolumn{12}{|l|}{ Hippocastanaceae } \\
\hline Aesculus hippocastanum L. & $\mathrm{EN}$ & EN & - & - & - & - & + & + & - & - & 4 \\
\hline \multicolumn{12}{|l|}{ Hypericaceae (Guttiferae) } \\
\hline Hypericum androsaemum L. & $\mathrm{R}$ & EN & - & - & - & - & - & + & - & - & 3 \\
\hline Hypericum calycinum L. & $\mathrm{R}$ & - & - & - & - & - & - & + & - & - & 2 \\
\hline \multicolumn{12}{|l|}{ Iridaceae } \\
\hline Crocus veluchensis Herbert & - & - & - & - & - & - & + & - & - & - & 1 \\
\hline \multicolumn{12}{|l|}{ Lamiaceae (Labiatae) } \\
\hline Marrubium friwaldskyanum Boiss. & $\mathrm{R}$ & VU & - & $\mathrm{R}$ & $\mathrm{R}$ & + & - & - & - & - & 4 \\
\hline Salvia forskahlei L. & EN & - & - & - & - & - & - & + & - & - & 2 \\
\hline Salvia pinnata $\mathrm{L}$. & - & $\mathrm{CR}$ & - & - & - & - & - & + & - & - & 2 \\
\hline Salvia ringens Sibth. \& Sm. & - & - & - & - & - & - & + & - & - & - & 1 \\
\hline
\end{tabular}




\begin{tabular}{|c|c|c|c|c|c|c|c|c|c|c|c|}
\hline 1 & 2 & 3 & 4 & 5 & 6 & 7 & 8 & 9 & $\mathbf{1 0}$ & 11 & 12 \\
\hline Salvia scabiosifolia Lam. & EN & $\mathrm{CR}$ & $\mathrm{R}$ & $\mathrm{R}$ & $\mathrm{R}$ & - & - & + & - & - & 5 \\
\hline Salvia verbenaca $\mathrm{L}$. & $\mathrm{R}$ & - & - & - & - & - & - & - & - & - & 1 \\
\hline Satureja rumelica Velen. & $\mathrm{R}$ & - & $\mathrm{R}$ & - & I & + & - & - & - & - & 4 \\
\hline Satureja pilosa Velen. & - & - & - & $\mathrm{nt}$ & - & - & + & - & - & - & 2 \\
\hline Teucrium botrys L. & - & - & - & - & - & - & - & + & - & - & 1 \\
\hline Teucrium lamiifolium D'Urv. & $\mathrm{R}$ & - & - & $\mathrm{R}$ & $\mathrm{R}$ & - & - & + & + & - & 4 \\
\hline Thymus albanus Heinr. Braun & - & - & - & - & - & - & + & - & - & - & 1 \\
\hline Thymus atticus Čelak. & - & - & - & - & - & - & + & - & - & - & 1 \\
\hline Thymus bracteosus Vis. ex Benth. & - & - & - & - & - & - & - & + & - & - & 1 \\
\hline Thymus comptus Friv. & - & - & - & - & - & - & + & - & - & - & 1 \\
\hline $\begin{array}{l}\text { Thymus longedentatus (Degen \& } \\
\text { Urum.) Ronniger }\end{array}$ & - & - & - & - & - & - & + & - & - & - & 1 \\
\hline Thymus perinicus (Velen.) Jalas & $\mathrm{R}$ & EN & - & - & - & + & - & + & - & - & 4 \\
\hline Thymus stojanovii Degen & - & $\mathrm{CR}$ & - & - & - & + & - & + & - & - & 3 \\
\hline \multicolumn{12}{|l|}{ Liliaceae } \\
\hline Fritillaria pontica Wahlenb. & $\mathrm{R}$ & - & $\mathrm{R}$ & - & - & - & - & + & - & - & 3 \\
\hline \multicolumn{12}{|l|}{ Menyathaceae } \\
\hline Menyanthes trifoliata $\mathrm{L}$. & EN & EN & - & - & - & - & - & - & - & - & 2 \\
\hline \multicolumn{12}{|l|}{ Morinaceae } \\
\hline Morina persica L. & $\mathrm{R}$ & - & - & - & - & - & - & + & - & - & 2 \\
\hline \multicolumn{12}{|l|}{ Orchidaceae } \\
\hline Platanthera bifolia (L.) Rich. & - & - & - & - & - & - & - & - & - & + & 1 \\
\hline \multicolumn{12}{|l|}{ Paeoniaceae } \\
\hline Paeonia mascula (L.) Mill. & EN & EN & - & - & - & - & - & + & - & - & 3 \\
\hline Paeonia tenuifolia $\mathrm{L}$. & EN & EN & - & - & - & - & - & + & + & - & 4 \\
\hline \multicolumn{12}{|l|}{ Polygalaceae } \\
\hline $\begin{array}{l}\text { Polygala acarnanica (Chodat) Koz. } \\
\text { et Petrova }\end{array}$ & $\mathrm{R}$ & - & - & - & - & - & + & + & - & - & 3 \\
\hline Polygala amarella Crantz & $\mathrm{R}$ & EN & - & - & - & - & - & + & - & - & 3 \\
\hline Polygala alpestris Reichenb. & - & EN & - & - & - & - & - & + & - & - & 2 \\
\hline Polygala carniolica Kern. & $\mathrm{R}$ & EN & - & - & - & - & + & - & - & - & 3 \\
\hline Polygala hospital Heuff. & $\mathrm{R}$ & - & - & - & - & - & - & - & - & - & 1 \\
\hline Polygala monspeliaca $\mathrm{L}$. & $\mathrm{R}$ & - & - & - & - & - & - & - & - & - & 1 \\
\hline Polygala rhodopea (Velen.) Janch. & $\mathrm{R}$ & - & - & - & - & - & + & - & - & - & 2 \\
\hline Polygala supina Schreb. & $\mathrm{R}$ & - & - & - & - & - & - & - & - & - & 1 \\
\hline \multicolumn{12}{|l|}{ Primulaceae } \\
\hline Primula deorum Velen. & $\mathrm{R}$ & VU & $\mathrm{R}$ & - & $\mathrm{nt}$ & + & - & + & + & - & 7 \\
\hline Primula frondosa Janka & $\mathrm{R}$ & EN & $\mathrm{R}$ & - & $\mathrm{R}$ & + & - & + & + & - & 7 \\
\hline Primula halleri G. F. Gmel. & $\mathrm{R}$ & - & - & - & - & - & - & + & - & - & 2 \\
\hline Primula vulgaris Huds. & $\mathrm{R}$ & - & - & - & - & - & - & + & - & - & 2 \\
\hline \multicolumn{12}{|l|}{ Ranunculaceae } \\
\hline Adonis microcarpa DC. & - & EN & - & - & - & - & - & - & - & - & 1 \\
\hline Adonis vernalis $\mathrm{L}$. & $\mathrm{R}$ & - & - & - & - & - & - & - & - & + & 2 \\
\hline Adonis wolgensis Stev. in DC. & $\mathrm{R}$ & - & - & - & - & - & - & + & - & - & 2 \\
\hline Anemone narcissiflora L. & $\mathrm{R}$ & - & - & - & - & - & - & + & - & - & 2 \\
\hline
\end{tabular}




\begin{tabular}{|c|c|c|c|c|c|c|c|c|c|c|c|}
\hline 1 & 2 & 3 & 4 & 5 & 6 & 7 & 8 & 9 & 10 & 11 & 12 \\
\hline Anemone sylvestris L. & $\mathrm{EN}$ & - & - & - & - & - & - & + & - & - & 2 \\
\hline Aquilegia aurea Janka & $\mathrm{R}$ & - & - & - & - & - & + & + & - & - & 3 \\
\hline Aquilegia nigricans Baumg. & $\mathrm{R}$ & - & - & - & - & - & + & + & - & - & 3 \\
\hline Caltha cornuta Schott & - & - & - & - & - & - & - & + & - & - & 1 \\
\hline Caltha polypetala Hochst. ex Lorent & - & EN & - & - & - & - & - & + & - & - & 2 \\
\hline Pulsatilla halleri (All.) Willd. & $\mathrm{R}$ & $\mathrm{EN}$ & - & - & - & - & - & - & + & - & 3 \\
\hline Pulsatilla pratensis (L.) Mill. & $\mathrm{R}$ & - & - & - & - & - & - & + & - & - & 2 \\
\hline Thalictrum foetidum $\mathrm{L}$. & $\mathrm{EN}$ & $\mathrm{CR}$ & - & - & - & - & - & + & - & - & 3 \\
\hline Trollius europaeus L. & $\mathrm{R}$ & - & - & - & - & - & - & + & - & - & 2 \\
\hline \multicolumn{12}{|l|}{ Rosaceae } \\
\hline Crataegus orientalis Pall. ex M. B. & - & - & - & - & - & - & - & + & - & - & 1 \\
\hline Mespilus germanica L. & $\mathrm{R}$ & - & - & - & - & - & - & - & - & - & 1 \\
\hline Potentilla chrysantha Trevir. & $\mathrm{R}$ & - & - & - & - & - & - & + & - & - & 2 \\
\hline Potentilla emili-popii Nyarady & $\mathrm{R}$ & $\mathrm{CR}$ & $\mathrm{R}$ & - & - & - & + & + & + & - & 6 \\
\hline Potentilla fruticosa L. & $\mathrm{R}$ & - & - & - & - & - & - & - & - & - & 1 \\
\hline Potentilla montenegrina Pant. & - & $\mathrm{CR}$ & - & - & - & - & - & + & - & - & 2 \\
\hline Potentilla nicicii Adam. & $\mathrm{R}$ & $\mathrm{CR}$ & - & - & - & - & - & + & - & - & 3 \\
\hline Potentilla pirotensis (Borb.) Mark. & - & - & - & - & - & - & + & - & - & - & 1 \\
\hline Potentilla palustris (L.) Scop. & $\mathrm{R}$ & - & - & - & - & - & - & + & - & - & 2 \\
\hline Potentilla regis-borisii Stoj. & - & - & - & - & - & - & + & - & - & - & 1 \\
\hline Pyracantha coccinea M. Roem. & EN & - & - & - & - & - & - & - & - & - & 1 \\
\hline Rubus macrophyllus Weihe \& Nees & EN & - & - & - & - & - & - & - & - & - & 1 \\
\hline Rubus oblongoobovatus Markova & $\mathrm{R}$ & - & - & - & - & + & - & - & - & - & 2 \\
\hline Rubus thyrsiflorus Wehe et Nees & EN & - & - & - & - & - & - & - & - & - & 1 \\
\hline Rubus vepallidus Sudre & $\mathrm{R}$ & - & - & - & - & - & - & - & - & - & 1 \\
\hline Spiraea crenata L. & $\mathrm{EN}$ & $\mathrm{CR}$ & - & E & $\mathrm{V}$ & - & - & + & - & - & 4 \\
\hline Spiraea hypericifolia L. & EN & - & - & - & - & - & - & - & - & - & 1 \\
\hline Spiraea salicifolia L. & EN & $\mathrm{CR}$ & - & - & - & - & - & + & - & - & 3 \\
\hline \multicolumn{12}{|l|}{ Rutaceae } \\
\hline Ruta graveolens L. & EN & EN & - & - & - & - & - & + & - & - & 3 \\
\hline \multicolumn{12}{|l|}{ Salicaceae } \\
\hline Salix pentandra L. & EN & $\mathrm{CR}$ & - & - & - & - & - & + & - & - & 3 \\
\hline Salix rosmarinifolia L. & - & $\mathrm{CR}$ & - & - & - & - & - & + & - & - & 2 \\
\hline \multicolumn{12}{|l|}{ Scrophulariaceae } \\
\hline Pedicularis palustris L. & $\mathrm{R}$ & $\mathrm{CR}$ & - & - & - & - & - & + & - & - & 3 \\
\hline Veronica austriaca L. & $\mathrm{R}$ & - & - & - & - & - & - & - & - & - & 1 \\
\hline Veronica chamaedrys L. & $\mathrm{R}$ & - & - & - & - & - & - & - & - & - & 1 \\
\hline \multicolumn{12}{|l|}{ Thymelaeaceae } \\
\hline Daphne laureola L. & $\mathrm{R}$ & $\mathrm{EN}$ & - & - & - & - & - & + & - & - & 3 \\
\hline Daphne pontica L. & $\mathrm{R}$ & $\mathrm{EN}$ & - & - & - & - & - & + & - & - & 3 \\
\hline \multicolumn{12}{|l|}{ Tiliaceae } \\
\hline Tilia rubra DC. & $\mathrm{R}$ & - & - & - & - & - & - & - & - & - & 1 \\
\hline \multicolumn{12}{|l|}{ Valerianaceae } \\
\hline Valeriana dioscoridis $\mathrm{Sm}$. & $\mathrm{R}$ & $\mathrm{EN}$ & - & - & - & - & - & - & - & - & 2 \\
\hline
\end{tabular}

* Column 12 represents the number of different documents (Red lists, Red data books and different legislative documents) where the respective taxon is included 
Eighty-one (8\%) species are protected by the Bulgarian legislation and they are included in the Appendix 3 of the Biodiversity Act from 2007.

Seventeen species (1,7\%) are in the "European List of Rare, Endangered and Endemic Plants" (Lucas 1983). It defines the National (BG) and European (EU) status of the species. According to the national status, 6 species are with category "Rare" (R), 3 species with category "Vulnerable" (VU), 2 species with category "Endangered" (E), 1 species with category "Undetermined" (I), and 2 species "neither Rare, nor Endangered". Accordingly to the European status 7 species are with category "Rare" (R), 3 species with category "Vulnerable" (V), 2 species with category "Undetermined", and 1 species with category "neither Rare, nor Endangered" (nt).

Eleven melliferous species fall in the IUCN's Red List of Endangered Species of 1997 (Walter and Gillett 1998). These are: Buglossoides grandulosa, Onosma rhodopea, Campanula lanata, C. transsilvanica, Chamaecytisus nejčeffii, Salvia scabiosifolia, Satureja rumelica, Fritillaria pontica, Primula deorum, P. frondosa, and Potentilla emili-popii.

Eight European melliferous species are strictly protected under the Bern Convention [ANONYMOUS, 1979], Appendix 2. These are: Campanula lanata, C. patula, Vaccinium arctostaphylos, Teucrium lamiifolium, Paeonia tenuifolia, Primula deorum, P. frondosa, Pulsatilla halleri, and Potentilla emili-popii Nyarady.

Four species are listed in Appendix 2 of the Convention on International Trade in Endangered Species [ANONYMOUS 1973, SUPPLEMENTED IN 2003], and these are: Galanthus elwesii, G. nivalis, Platanthera bifolia and Adonis vernalis L.

Thirty three species $(3,3 \%)$ among the melliferous plants are Bulgarian endemics and 54 $(5,3 \%)$ are Balkan endemics.

\section{Conclusion}

The data presented so far show that the melliferous plants of the Bulgarian flora amount 201 species from 71 genera and 36 families. This represents $20 \%$ of the species, $24,3 \%$ of the genera and $42,9 \%$ of the families with wild melliferous plants. With the highest conservation status among them are the Bulgarian endemics Primula deorum and P. frondosa, included in seven lists of conservation importance. They are followed by Onosma rhodopea, Potentilla emili-popii - present on six lists, Campanula lanata, C. transsilvanica, Chamaecytisus kovačevii, C. neičeffii, Salvia scabiosifolia, Vaccinium arctostaphylos - part of 5 lists, and Aesculus hippocastanum, Buglossoides grandulosa, Erodium absinthoides, Galanthus elwesii, G. nivalis, Marrubium friwaldskyanum, Matthiola odoratissima, Paeonia tenuifolia, Satureja rumelica, Spiraea crenata, Teucrium lamiifolium, Thymus perinicus, which are part of 4 list.

The results outline the high conservation importance of the Bulgarian melliferous plants, both for the national and for the European flora.

\section{References}

ANONYMOUS, 1979. Convention on the Conservation of European Wildlife and Natural Habitats. (http://conventions.coe.int/treaty/en/treaties/html/104.htm)

ANONYMOUS, 1998. Convention on International Trade in Endangered Species (CITES) 1998. (http://www.cites.org/eng/app/appendices.php)

Anonymous, 2002. Biodiversity Act of Bulgaria. State Gazette, 77/2002, with amendments up to 2011. (http://lex.bg/laws/ldoc/2135456926) (in Bulgarian)

Assyov B., Petrova A. (eds). Conspectus of the vascular flora of Bulgaria. Chorology and floristic elements. Bulgarian Biodiversity Foundation, Sofia, 2006. - 454 p. (in Bulgarian)

LUCAS G. List of Rare Threatened and Endemic Plants in Europe. - Strasburg, 1983. - 358 p.

Peev D. (ed.). Red Data Book of Bulgaria. Vol.1. Plants and Fungi. - Bulg. Acad. of Sci. Publ. House, Sofia, (in press).

Petrova A., Vladimirov V. (eds) Red List of Bulgarian vascular plants // Phytologia Balcanica. - 2009. Vol. 15,N 1. - P. 63-94.

TASHEV A. Flora of the vascular plants of Bulgaria: history of studies, structure and problems of conservation // Ukr. Bot. Zhurnal. - 2008. - Vol. 65, N 1. - P. 41-57. (in Russian). 
Tashev A., Pancheva E. Honey bearing plants of Bulgaria / Modern problems of the botany and ecology. International scientific conference of young scientists (21-25.09.2010). - Yalta-Simferopol: "Arial”? 2010. - P. 140-142.

VelcheV V. (ed.) Red Data Book of Bulgaria. Vol. I., Plants. - Bulg. Acad. of Sci. Publ. House, Sofia, 1984. $448 \mathrm{p}$.

Walter K. S., Gillett H. J. (eds). 1997 IUCN Red List of Threatened Plants. Compiled by the World Conservation Monitoring Centre. IUCN - The World Conservation Union. Gland, Switzerland and Cambridge, UK. - 1998. - LXIV + 862 p.

Рекомендує до друку

Отримано 10.08.2011 p.

А.В. Сна

Aдреса авторів:

О. Тамев, Е. Панчева

Університет лісу,

Софія, Болгарія

E-mail: atashev@mail.bg,jeni_pancheva@abv.bg
Author's address:

Alexander Tashev

Evgenia Pancheva

University of Forestry,

Sofia, Bulgaria

E-mail: atashev@mail.bg,jeni_pancheva@abv.bg 